\title{
Grass root destruction
}

\section{Peter D. Moore}

THE consumption of plant material by invertebrate herbivores can represent a considerable energetic loss for a plant. For example, in a study of the willow Salix cinerea in North Wales, 45 per cent of leaves were found to be damaged and roughly 13 per cent of the leaf area of the tree had been lost to invertebrate feeders ${ }^{1}$; for smaller herbaceous plants subjected to heavy insect feeding pressure in grasslands, losses may prove even more serious. The long-term damage inflicted by insects on plant populations is assessed in two recent reports by Brown and Gange in Oikos $^{2}$ and in Functional Ecology ${ }^{3}$. Because selective consumption by insects results in severely reduced plant size, insect herbivory can increase the variability in individual shoot size in a population below ground; invertebrate grazers may further modify the composition of a plant community, and even influence its species diversity $^{3}$.

Because of the complexity of plantinvertebrate interactions, many studies in this area have concentrated on single plant species and their response to a particular species of herbivorous invertebrate. Often such work is related to pest problems, or the possibilities of using plantfeeding insects for weed control ${ }^{4}$. But there are broader questions in community ecology that cannot be answered by such specific experiments. For example, what are the general effects of insect herbivory on a plant community and on the development of successions? If such questions were applied to mammalian grazers, the experimental technique that would spring to mind for their solution is the exclusion of the grazers from a sample plot and the observation of consequent changes in vegetation structure and composition in comparison with grazed areas. This experimental design is not so easily achieved for invertebrates, but at the Silwood Park field station of Imperial College, London, a series of long-term experiments has been initiated in which insecticides are sprayed on grassland plots from which the insects are to be excluded. These plots are selected to represent various stages in successional development, from one to 16 years.

Gange and Brown ${ }^{2}$ describe the results from one experiment in which they investigated the influence of invertebrate herbivory on individual plant size. They found that plant size within eight species examined is generally greater in those plots where insecticide has been applied. There is also a smaller range in plant size in treated plots. So insect herbivory, by selectively damaging sensitive individuals, produces a skewed size frequency distri- bution among the plants. The influence of herbivory is thus operating in a manner similar to competition in that the less fit (that is, more palatable) individuals are reduced in size and thus placed at a disadvantage. Annual species respond rapidly, but in perennial species the effect is cumulative: loss of this year's photosynthetic tissue will affect next year's productivity. For this reason, size variability within a plant species becomes greater as succession proceeds.

Meanwhile, below the surface of the ground, root herbivory also takes its toll on plant tissues. This can be studied by similar methods to shoot herbivory, but using soil insecticides instead ${ }^{3}$. In this case, one of the most apparent effects on the plant community of removing soil insects is an increase in the number of plant species. Over a three-year succession from open ground, 83 species were recorded in treated plots compared with 70 in the controls. It would seem, therefore, that subterranean grazing is preventing the establishment of certain plant species. Brown and Gange point out that this constitutes an apparent contradiction of Grime's model ${ }^{5}$, in which above-ground grazing pressures increase diversity by reducing the dominance of robust plant species. But the early successional stage used in the Silwood Park experiment (up to three years) introduced factors of disturbance and a lack of stability into the equation, that may make this comparison invalid.

What is evident, however, is that soil herbivory must be taken into account in studies of community development. This fact has been further underlined by findings in the Chihuahuan Desert in New Mexico $^{6}$, where changes in the spatial distribution of the mesquite shrub (Prosopis glandulosa) in the past hundred years could be due to the intensity of attack by nematodes in the soil. In playa and arroyo habitats, in which the mesquite is becoming increasingly scarce, nematodes have been found down to depths of 12 metres, feeding upon even the deepest roots. Grassland and dune habitats are being invaded by mesquite, and here the depth to which nematodes penetrate is much smaller (less then 3 metres).

The study of what goes on below ground is proving an important development area in ecology, not only in the investigation of plant productivity and carbon sinks ${ }^{7}$, but also in the understanding of what controls the composition and successional development of plant communities.

Peter D. Moore is in the Division of Biosphere Sciences, King's College, University of London, Campden Hill Road, London W8 7AH, UK.

1. Alliende, M.C. J. Ecol. 77, 1048-1058 (1989).

2. Gange, A.C. \& Brown, V.K. Oikos 56, 351-356 (1989)

3. Brown, V.K. \& Gange, A.C. Funct. Ecol. 3, 667-671 (1989).

4. Moran, V.C. \& Hoffman, J.H. J. appl. Ecol. 26, 967-977 (1989).

5. Grime, J.P. Plant Strategies and Vegetation Processes (Wiley, Chichester, 1979)

Freckman, D.W. \& Virginea, R.A. Ecology 70, 1665-1678 (1989).

7. Moore, P.D. Nature 348, 858 (1989)

\section{Effects of CFC substitutes}

\section{Keith Shine}

THE ratification in 1988 of the Montreal Protocol on protecting the ozone layer ${ }^{1.2}$ and the increasing pressure for its controls to be strengthened has naturally accelerated the search for potential substitutes to chlorofluorocarbons (CFCs). Although some companies are opting for non-halogenated molecules, much interest is being shown in a family of closely related molecules known generally as the hydrohalocarbons. No longer is there a presumption that such gases in the atmosphere are innocent until proven guilty and, in parallel with toxicity tests, their possible environmental effects are now being examined. The results of the first detailed study of the possible effects of the CFC substitutes on the ozone layer and greenhouse warming are reported in the two papers by Fisher et al. on pages 508 and 513 of this issue $e^{3.4}$.

The so-called hard CFCs (CFC-11, -12 , $-113,-114$ and -115 ) have the dubious priv- ilege of playing important roles in both ozone depletion and greenhouse warming. The signing of the Montreal Protocol led to hopes that measures aimed at protecting the ozone layer would also lead to a reduction in the predicted warming. Wigley $^{5}$ showed that adherence to the protocol could reduce the warming effects of the CFCs to between one-fifth and one-seventh of pre-Protocol projections. Wigley also considered a pessimistic picture in which the reductions in CFC-11 and CFC-12 emissions were made up for by emissions of one of the hydrochlorofluorocarbons, HCFC-22, a substitute already in widespread use; the reduction due to CFCs was four times greater than the increase due to HCFC22. But more recent spectroscopic measurements have shown that the greenhouse strength of HCFC-22 was severely underestimated in earlier studies $^{6.7}$; an analysis similar to Wigley's, but 